\title{
Complete and Durable Remission of Human Epidermal Growth Factor Receptor 2-Positive Metastatic Urothelial Carcinoma Following Third-Line Treatment with Trastuzumab and Gemcitabine
}

\author{
Felix Wezel $^{\mathrm{a}}$ b Philipp Erben $^{\mathrm{b}}$ Timo Gaiser ${ }^{\mathrm{c}}$ Johannes Budjan ${ }^{\mathrm{d}}$ \\ Jost von Hardenberg ${ }^{b}$ Maurice Stephan Michel $^{b}$ Christian Bolenz ${ }^{a, b}$ \\ ${ }^{a}$ Department of Urology, University of Ulm, Ulm, ${ }^{b}$ Department of Urology, ${ }^{c}$ Institute of Pathology and ${ }^{\mathrm{d}}$ Institute of Clinical \\ Radiology and Nuclear Medicine, University Medical Center Mannheim, Mannheim, Germany
}

\section{Key Words}

Human epidermal growth factor receptor 2 - ERBB2 gene .

Urothelial cancer $\cdot$ Bladder $\cdot$ Trastuzumab

\begin{abstract}
Urothelial carcinoma (UC) is one of the most common cancers and survival rates are low in metastatic disease with currently established first-line platinum-based chemotherapies. Unlike in many other cancers, no clinically established molecular targeted therapies exist for the treatment of this malignancy. Here we present a case of complete tumor remission following third-line treatment with trastuzumab and gemcitabine in a patient with human epidermal growth factor receptor 2 (HER2)-positive UC after progression under cisplatin and vinflunine chemotherapies. This case shows the potential significance of anti-HER2 therapy in selected patients with molecularly characterized UC. Clinical trials so far show inconclusive outcomes of anti-HER2 therapies in UC, indicating further need for both basic research and clinical studies for the identification of resistance factors and improved patient selection.

(c) 2016 S. Karger AG, Basel
\end{abstract}

\section{Introduction}

Patients with metastatic urothelial carcinoma (UC) show poor response rates to platinum-based chemotherapy as the current standard treatment of care, resulting in a median survival of about 14 months [1]. Vinflunine is a second-line treatment option but offers only a slight survival benefit compared to best supportive care [2]. In contrast to many other cancer entities, there are currently no clinically established molecularly driven 'precision medicine' treatment approaches targeting potential oncogenes in UC. Previous reports have suggested that human epidermal growth factor receptor 2 (HER2) positivity in UC may predict response to trastuzumab, which is an established targeted treatment in metastatic breast cancer [3]. Here we present a case report of a patient who achieved complete remission after third-line treatment with trastuzumab and gemcitabine in a HER2 expressing metastatic UC.

\section{Clinical Course}

The patient was diagnosed with a pT1 G2 UC of the left distal ureter in 2006 and underwent resection of the distal ureter. In 2009, the patient presented with radio-

\section{KARGER}

(C) 2016 S. Karger AG, Basel

E-Mail karger@karger.com

www.karger.com/uin
Dr. med. Felix Wezel, MSc

Department of Urology

University Hospital Ulm

Prittwitzstr. 43, DE-89075 Ulm (Germany)

E-Mail felix.wezel@uniklinik-ulm.de 

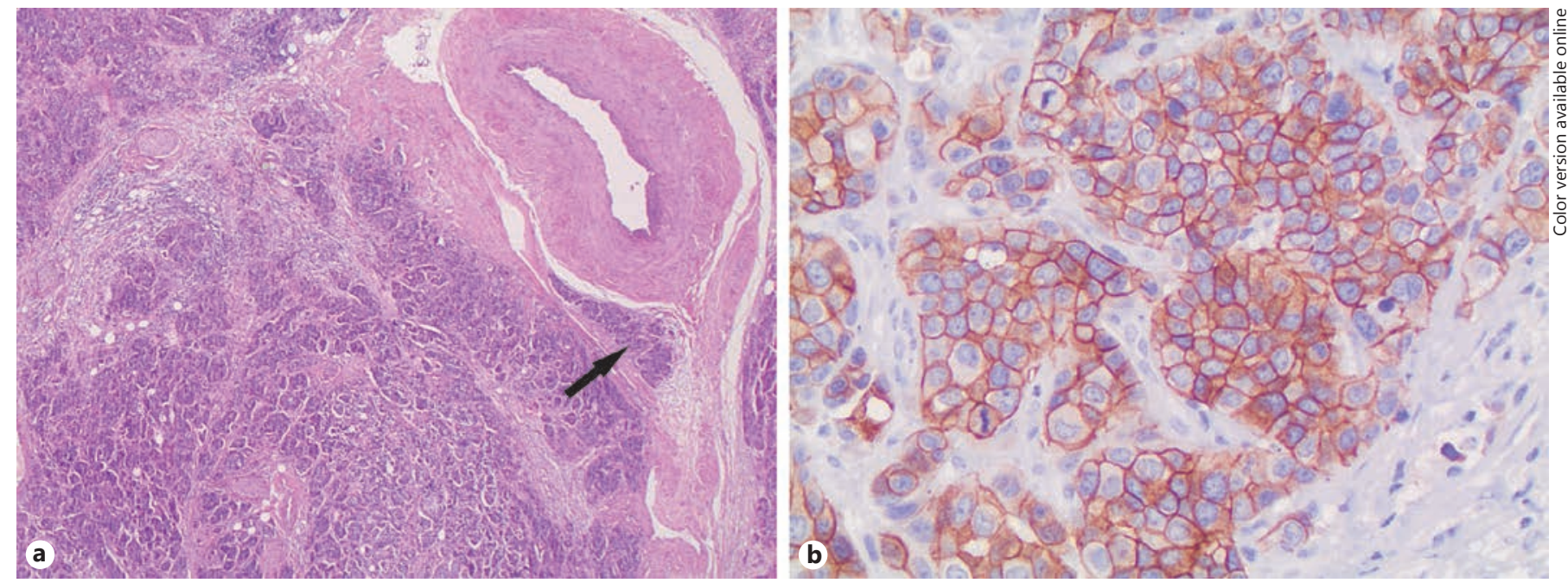

Fig. 1. a Haematoxylin-Eosin staining shows the locally advanced UC infiltrating the spermatic duct (arrow) (25×). b Immunolabelling revealed strong HER2 positivity (IHC 3+, c-erb-2; A0485, Dako) (200×).

logically confirmed disease recurrence with a retroperitoneal tumor mass and consecutive hydronephrosis. Surgical exploration revealed a non-resectable T4 disease with infiltration of the rectum and metastasis in regional lymph nodes and peritoneum. An inductive chemotherapy with four cycles of cisplatin with gemcitabine was applied and this treatment method resulted in partial remission. The patient continued with radiochemotherapy with 54 Gy and 6 cycles of cisplatin. Two years later, tumor recurrence was detected retroperitoneally and in the spine. The patient therefore received palliative radiochemotherapy including 6 cycles of cisplatin. During followup, the patient developed progressive disease with retroperitoneal lymph node metastasis and subsequently received second-line chemotherapy with vinflunine (320 $\mathrm{mg} / \mathrm{m}^{2}$ ); however, re-staging demonstrated progression after 3 cycles. As a last resort, biomarker testing of the primary tumor was applied and this revealed a strong immunohistochemical HER2 expression (fig. 1). However, fluorescence in situ hybridization (FISH) showed no HER2 gene amplification.

The patient was offered an experimental third-line treatment with trastuzumab $(6 \mathrm{mg} / \mathrm{kg} 3$-weekly; loading dose $8 \mathrm{mg} / \mathrm{kg})$ in combination with gemcitabine $(1,000$ $\mathrm{mg} / \mathrm{m}^{2}$ body surface area). Significant regression of the metastatic lesions was apparent after four cycles of trastuzumab and gemcitabine. After another four treatment cycles, MRI showed complete tumor remission without detectable metastatic lesions (fig. 2) so that the therapy was discontinued. The patient reported no toxicities and the clinical course has been uneventful for over 12 months now since induction and 5 months after cessation of trastuzumab/gemcitabine treatment.

\section{Discussion}

Current treatment protocols for metastatic UC offer platinum-based chemotherapy as first-line treatment with limited response rates [1]. Vinflunine may be applied as second-line chemotherapy with marginal survival benefits compared to best supportive care [2]. Despite growing evidence that molecularly driven targeted therapies might be effective in UC, no established and clinically approved treatments are currently available. Here we report a case of complete and durable remission of metastatic UC following third-line treatment with trastuzumab and gemcitabine in a HER2 expressing primary tumor, thus indicating a potential role of anti-HER2 therapy in a subset of patients.

Targeting the oncogene HER2 with the humanized antibody trastuzumab is a well-established treatment in breast cancer where HER2 is overexpressed in approximately $20 \%$ of patients [3]. A recent study has evaluated gene amplification and expression status by FISH and IHC in a large cohort of more than 37,000 tumors, in which UC was the most frequently HER2 expressing tumor type of all tested solid tumors [4]. Among 475 examined UC samples, $12.4 \%$ strongly expressed HER2 (IHC $3+$ ) and IHC correlated in $>90 \%$ with positive 

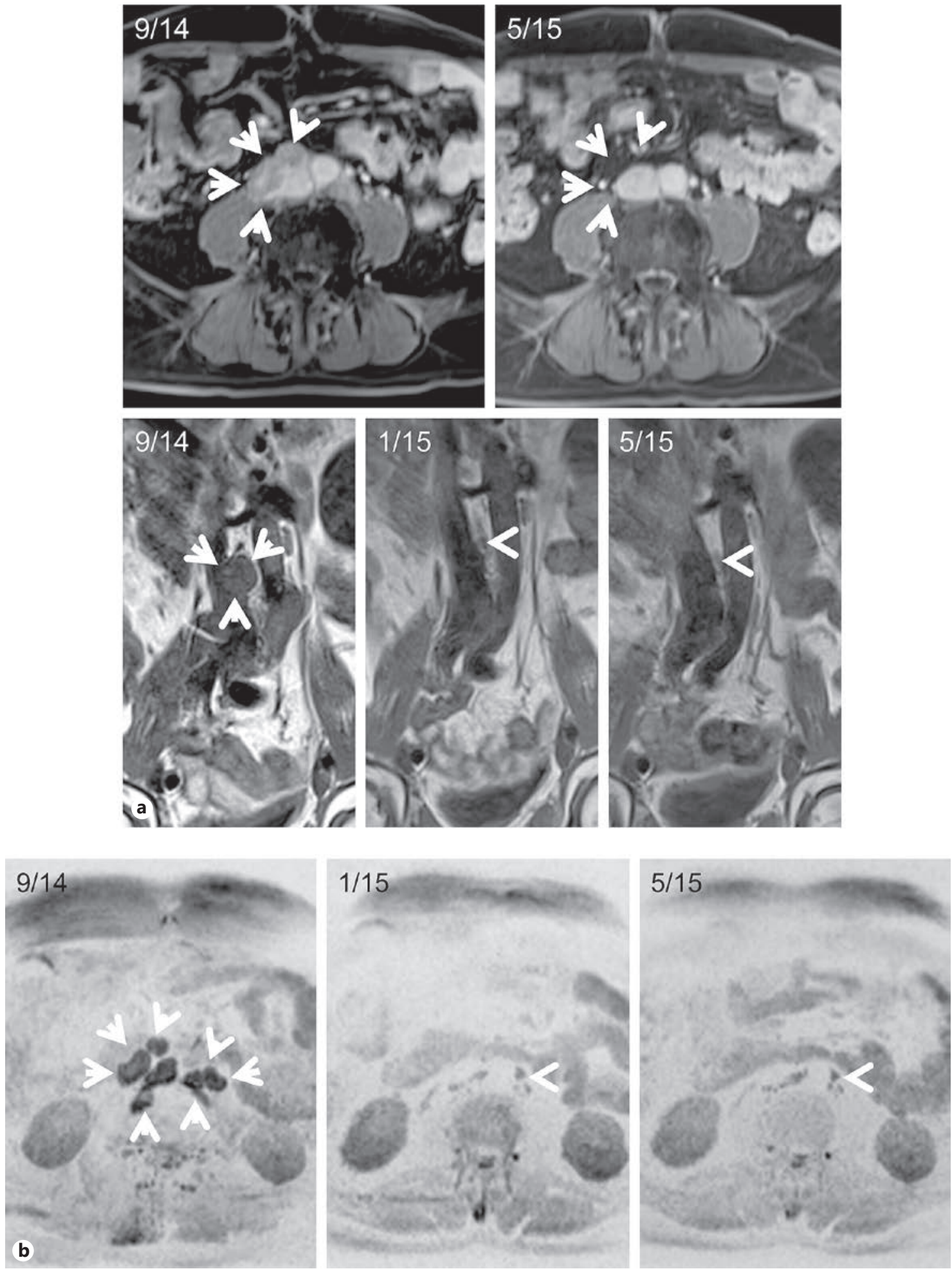

Fig. 2. a Enlarged interaortocaval nodal masses (arrows) prior therapy. Regression during therapy with only residual lymph nodes 5/15 (upper row: axial T1 weighted, post contrast image with fat suppression; lower row: coronal T1 weighted images pre con-

trast). b Enlarged retroperitoneal nodal masses (arrows) prior therapy (left). Regression during therapy with only residual lymph nodes $1 / 15$ and $5 / 15$ (axial diffusion weighted imaging, $b=800 \mathrm{~s} /$ $\mathrm{mm}^{2}$, inverted colors, maximum intensity projection). 
FISH. Previous reports have demonstrated that HER2 expression in UC correlates with aggressive disease and poor survival rates $[5,6]$. However, the two currently existing clinical phase II trials assessing the efficacy of trastuzumab-enhanced treatment in HER2-positive UC yielded rather inconclusive outcomes $[7,8]$. Hussain et al. [7] investigated a new combination of trastuzumab with carboplatin, paclitaxel and gemcitabine in 44 HER2positive patients. Twenty five (57\%) of 44 patients had a confirmed response and the median time to progression and survival were 9.3 and 14.1 months, respectively. No updated survival data have been reported yet. In a recently published randomized phase II multicenter trial, Oudard et al. [8] investigated platinum-based chemotherapy with or without trastuzumab in HER2-positive UC. In a cohort of 563 patients, the authors identified 75 (13.3\%) HER2-positive (IHC 2+/3+ and FISH+) primary tumors and finally included 61 patients in the study. Interestingly, there was no significant difference between the two treatment arms regarding response and overall survival rates (15.7 months without trastuzumab vs. 14.1 months with trastuzumab). Although the true benefit of trastuzumab might be masked by the simultaneous addition of first-line cytotoxic agents, the Oudard trial [8] suggests that current diagnostic work up might be insufficient for selecting the right patient. Discrepancies exist regarding the rates of HER2-positive patients between different reports although standardized IHC tests are available [5]. Controversy also surrounds the significance of IHC and FISH concordance [9] and our presented case confirms that absent gene amplification evaluated by FISH does not preclude clinical response to anti-HER2 therapy when HER2 protein is strongly expressed. Furthermore, in the individual case, it is unclear if metastatic lesions share the same HER2 expression pattern compared to the primary tumor. Although high correlations have been described in initially resected lymph nodes, little is known about recurrent disease patterns in pretreated tumors $[5,6]$. Recent advances in the molecular understanding of HER2-amplified cancers have helped to understand intrinsic resistance mechanisms to HER2 targeting drugs. This includes, for example, the presence of truncated HER2 isoforms lacking the binding region for trastuzumab, activating HER2 mutations that maintain downstream signaling despite extracellular HER2 inhibition or induction of alternative oncogenic pathways such as PI3K or MET to bypass HER2-dependent signaling (reviewed in [10]). In UC, neither the expression of alternative HER2 isoforms nor the spectrum of prevalent HER2 mutations are well defined and may explain the apparent failure in the Oudard trial [8]. Our presented case, however, demonstrates that HER2 targeting is a potentially powerful weapon in a subset of patients with metastatic UC but the treatment may have to be tailored more closely to the individual molecular setting.

\section{References}

1 von der Maase H, Sengelov L, Roberts JT, Ricci S, Dogliotti L, Oliver T, et al: Long-term survival results of a randomized trial comparing gemcitabine plus cisplatin, with methotrexate, vinblastine, doxorubicin, plus cisplatin in patients with bladder cancer. J Clin Oncol 2005;23:4602-4608.

2 Bellmunt J, Théodore C, Demkov T, Komyakov B, Sengelov L, Daugaard G, et al: Phase III trial of vinflunine plus best supportive care compared with best supportive care alone after a platinum-containing regimen in patients with advanced transitional cell carcinoma of the urothelial tract. J Clin Oncol 2009;27: 4454-4461.

3 Rimawi MF, Schiff R, Osborne CK: Targeting HER2 for the treatment of breast cancer. Annu Rev Med 2015;66:111-128.

4 Yan M, Schwaederle M, Arguello D, Millis SZ, Gatalica Z, Kurzrock R: HER2 expression sta- tus in diverse cancers: review of results from 37,992 patients. Cancer Metastasis Rev 2015; 34:157-164.

5 Bolenz C, Shariat SF, Karakiewicz PI, Ashfaq R, Ho R, Sagalowsky AI, et al: Human epidermal growth factor receptor 2 expression status provides independent prognostic information in patients with urothelial carcinoma of the urinary bladder. BJU Int 2010;106:12161222.

6 Fleischmann A, Rotzer D, Seiler R, Studer UE, Thalmann GN: HER2 amplification is significantly more frequent in lymph node metastases from urothelial bladder cancer than in the primary tumours. Eur Urol 2011; 60:350-357.

7 Hussain MH, MacVicar GR, Petrylak DP, Dunn RL, Vaishampayan U, Lara PN Jr, et al: Trastuzumab, paclitaxel, carboplatin, and gemcitabine in advanced human epidermal growth factor receptor-2/neu-positive urothelial carcinoma: results of a multicenter phase II national cancer institute trial. J Clin Oncol 2007;25:2218-2224.

8 Oudard S, Culine S, Vano Y, Goldwasser F, Théodore C, Nguyen T, et al: Multicentre randomised phase II trial of gemcitabine + platinum, with or without trastuzumab, in advanced or metastatic urothelial carcinoma overexpressing HER2. Eur J Cancer 2015;51: 45-54.

9 Coogan CL, Estrada CR, Kapur S, Bloom KJ HER-2/neu protein overexpression and gene amplification in human transitional cell carcinoma of the bladder. Urology 2004;63:786790.

10 Arteaga CL, Engelman JA: ERBB receptors: from oncogene discovery to basic science to mechanism-based cancer therapeutics. Cancer Cell 2014;25:282-303. 